\title{
Effects of resveratrol and genistein on nuclear factor- $\kappa B$, tumor necrosis factor- $\alpha$ and matrix metalloproteinase-9 in patients with chronic obstructive pulmonary disease
}

\author{
XIAO-JU LIU, HAI-RONG BAO, XIAO-LI ZENG and JUN-MING WEI
}

Department of Gerontal Respiratory Medicine, The First Hospital of Lanzhou University, Lanzhou, Gansu 730000, P.R. China

Received June 22, 2015; Accepted March 10, 2016

DOI: $10.3892 / \mathrm{mmr} .2016 .5057$

\begin{abstract}
Chronic airway inflammation and airway remodeling are the major pathophysiological characteristics of chronic obstructive pulmonary disease (COPD). Resveratrol and genistein have been previously demonstrated to have anti-inflammatory and antioxidative properties. The present study aimed to measure the inhibitory effects of resveratrol and genistein on tumor necrosis factor (TNF)- $\alpha$ and matrix metalloproteinase (MMP)-9 concentration in patients with COPD. Lymphocytes were isolated from the blood of 34 patients with COPD and 30 healthy subjects, then randomly divided into the following four treatment groups: Control, dexamethasone $(0.5 \mu \mathrm{mol} / \mathrm{l})$, resveratrol $(12.5 \mu \mathrm{mol} / \mathrm{l})$ and genistein $(25 \mu \mathrm{mol} / \mathrm{l})$ groups. After $1 \mathrm{~h}$ of treatment, $100 \mu \mathrm{l}$ lymphocytes were collected for nuclear factor (NF)- $\mathrm{kB}$ immunocytochemical staining. After $48 \mathrm{~h}$ treatment, the supernatant of the lymphocytes was collected for analysis of TNF- $\alpha$ and MMP-9 concentration levels. The percentage of lymphocytes with positive nuclear NF- $\mathrm{KB}$ expression was analyzed by immunocytochemical staining. The concentration levels of TNF- $\alpha$ and MMP-9 were measured using radioimmunoassay and enzyme-linked immunosorbent assay, respectively. The present study demonstrated that the percentage of NF- $\kappa B-$ positive cells, and the levels of TNF- $\alpha$ and MMP-9 in lymphocytes from patients with COPD patients were significantly higher compared with healthy subjects. Additionally, there were positive correlations between the percentage of NF- $\mathrm{kB}-$ positive cells, and the concentration levels of TNF- $\alpha$ and MMP-9 in patients with COPD. All three factors were significantly reduced in lymphocytes treated with resveratrol and genistein, and the inhibitory effects of resveratrol on
\end{abstract}

Correspondence to: Professor Xiao-Ju Liu, Department of Gerontal Respiratory Medicine, The First Hospital of Lanzhou University, 1 Dong Gang West Road, Lanzhou, Gansu 730000, P.R. China

E-mail: liuxiaoju835@126.com

Key words: pulmonary disease, chronic obstructive, resveratrol, genistein, nuclear factor- $\kappa \mathrm{B}$, matrix metalloproteinase-9, tumor necrosis factor- $\alpha$
NF- $\kappa B, T N F-\alpha$ and MMP-9 were more potent than the effects of genistein. In conclusion, resveratrol and genistein may inhibit the NF- $\mathrm{BB}, \mathrm{TNF}-\alpha$ and MMP-9-associated pathways in patients with COPD. It is suggested that resveratrol and genistein may be potential drugs candidates for use in the treatment of COPD.

\section{Introduction}

Chronic obstructive pulmonary disease (COPD) is characterized by reduced airflow, which is typically progressive, and associated with an abnormal inflammatory response of the lungs to noxious particles and gases. The pathogenesis of COPD involves several processes, including pulmonary and systemic inflammation, protease/anti-protease imbalance, vascular and extracellular matrix (ECM) remodeling, and tissue repair $(1,2)$. Previous research reported that increased levels of macrophages, neutrophils and cytotoxic $\left(\mathrm{CD}^{+}\right) \mathrm{T}$ lymphocytes were recruited into airway walls during COPD; additionally, numerous proinflammatory mediators and cytokines were observed in the airway $(1,3,4)$. Nuclear factor $(N F)-\kappa B$ is a nuclear transcription factor. Following an inflammatory stimulus, phosphorylation of inhibitor of $\kappa \mathrm{B}$ (IкB) triggers its degradation and the translocation of NF- $\mathrm{KB}$ to the nucleus, where it induces the expression of a variety of genes, including cytokines [interleukin (IL)-1 $\beta$, IL- 6 and tumor necrosis factor (TNF)- $\alpha$ ], enzymes [cyclooxygenase- 2 and matrix metalloproteinases (MMPs)], adhesion molecules and acute phase proteins (5). IL-6 and TNF- $\alpha$ are critical proinflammatory factors, and MMPs are closely associated with lung injury and repair in patients with COPD. The functions of these inflammatory factors and MMPs are dependent on each other. The expression and activity of MMP-9 (also termed gelatinase B) can be induced by TNF- $\alpha$, with its induction potentially associated with the NF- $\mathrm{BB}$-mediated signal transduction pathway. TNF- $\alpha$-induced MMP-9 activity is suppressed by $18 \beta$-glycyrrhetinic acid via inhibition of the Akt-dependent NF- $\kappa B$ pathway (6). Resveratrol (trans-3,4',5-trihydroxystilbene), a natural polyphenol, is present in various plants, nuts and fruits. It is particularly abundant in grapes and red wine. Numerous previous studies have demonstrated that resveratrol has a wide range of pharmacological properties; for example, there is considerable evidence demonstrating that resveratrol has anti-inflammatory properties $(1,7,8)$, with 
one study observing that resveratrol attenuates lipopolysaccharide (LPS)-induced acute kidney injury by suppressing inflammation driven by macrophages (9). Resveratrol also has a therapeutic effect on house dust mite-induced mouse allergic asthma and its mechanism of action may be associated with reducing the production of spleen tyrosine kinase protein (10). Additionally, resveratrol has been demonstrated to protect against brain injury by downregulation of MMP-9 (11). An isoflavone compound, genistein (4',5,7-trihydroxyisoflavone), has been previously shown to act as a broad-spectrum tyrosine kinase inhibitor. Genistein possesses anti-inflammatory properties and, therefore, may be important in modulating mammalian inflammation pathways to inhibit certain types of chronic disease (12-14). Previous research revealed that genistein inhibits the activation of $\mathrm{NF}-\kappa \mathrm{B}$ and the production of TNF- $\alpha$ in patients with asthma (15). Although these natural compounds, including resveratrol and genistein, may reduce chronic inflammation, their action on the inflammatory mediators NF- $\kappa$ B, TNF- $\alpha$ and MMP-9 in lymphocytes from patients with COPD has not been fully elucidated. The present study aimed to observe the effects of resveratrol and genistein on the expression levels of NF- $\kappa$, TNF- $\alpha$ and MMP-9 in patients with COPD.

\section{Materials and methods}

Patients. Patients with COPD ( $\mathrm{n}=34$; age, $71.8 \pm 9.0$ years) were recruited from the Department of Gerontal Respiratory Medicine of the First Hospital of Lanzhou University (Lanzhou, China). No patients had received any medication for $24 \mathrm{~h}$ or any steroids for 2 weeks prior to collection of the $10 \mathrm{ml}$ blood sample by venepuncture into a heparinized tube for immediate use. The following criteria were required for inclusion in the present study: i) COPD confirmed by clinical history and abnormal lung function according to the criteria established by the Global Initiative for Chronic Obstructive Lung Disease guidelines (16); ii) COPD diagnosis $\geq 2$ years with $\geq 2$ exacerbations; and iii) no long-term maintenance therapy, with the exception of the inhalation of a short-acting bronchodilator as required. Patients with a history or diagnosis of asthma, lung cancer, bronchiectasis or severe heart disease were excluded from the study. Healthy subjects were defined as having normal lung function and no hypertension, diabetes mellitus, heart disease or similar conditions. The age and gender distribution of healthy subjects $(n=30)$ was similar to those of the patients with COPD (Table I). According to the ethical guidelines of the Helsinki Declaration, informed consent was obtained from all participants and was approved by the Ethical Committee of the First Hospital of Lanzhou University.

Lymphocyte preparation and experimental groups. Lymphocytes were isolated using Ficoll-Hypaque separation. Briefly, full blood was layered on Ficoll-Hypaque cushions (Beijing Solarbio Science and Technology Co., Ltd., Beijing, China) and centrifuged for $20 \mathrm{~min}$ at $1,000 \mathrm{x}$ g. Lymphocytes were collected from the interface, washed twice with phosphate-buffered saline (PBS; Beijing Solarbio Science and Technology Co., Ltd.) and suspended in complete RPMI-1640 medium (Thermo Fisher Scientific, Inc., Waltham, MA, USA) supplemented with $10 \%$ fetal calf serum (Thermo Fisher Scientific, Inc.), 2 mM
Table I. Demographic and clinical characteristics of patients with COPD $(n=34)$ and healthy subjects $(n=30)$.

\begin{tabular}{lcc}
\hline Characteristic & $\begin{array}{c}\text { COPD } \\
\text { patients }\end{array}$ & $\begin{array}{c}\text { Healthy } \\
\text { subjects }\end{array}$ \\
\hline Age, years & $71.8 \pm 9.0$ & $67.8 \pm 8.2$ \\
Gender (male/female), $\mathrm{n}$ & $23 / 11$ & $20 / 10$ \\
Smokers, n & 20 & 17 \\
$\mathrm{FEV}_{1}, \%$ & $52.16 \pm 24.07^{\mathrm{a}}$ & $104.10 \pm 14.73$ \\
$\mathrm{FEV}_{1} / \mathrm{FVC}, \%$ & $53.93 \pm 13.66^{\mathrm{a}}$ & $81.03 \pm 7.00$ \\
\hline
\end{tabular}

${ }^{\mathrm{a}} \mathrm{P}<0.01$ vs. healthy subjects. Age, $\mathrm{FEV}_{1}$ and $\mathrm{FVC}$ data are presented as the mean \pm standard deviation. COPD, chronic obstructive pulmonary disease; $\mathrm{FEV}_{1}$, forced expiratory volume in $1 \mathrm{sec} ; \mathrm{FVC}$, forced vital capacity.

L-glutamine (Beijing Solarbio Science and Technology Co., Ltd.), $100 \mathrm{IU} / \mathrm{ml}$ penicillin and $100 \mu \mathrm{g} / \mathrm{ml}$ streptomycin (North China Pharmaceutical Group Corporation, Shijiazhuang, China), at a concentration of $2 \times 10^{6}$ cells $/ \mathrm{ml}$. The viability of lymphocytes was determined using trypan blue (Invitrogen; Thermo Fisher Scientific, Inc., Waltham, MA, USA) exclusion assay (17). Viable lymphocytes were seeded into 24-well culture plates and were maintained in a humidified incubator at $37^{\circ} \mathrm{C}$ and a $5 \% \mathrm{CO}_{2}$ atmosphere. Lymphocytes from patients with COPD and healthy subjects were randomly divided into four groups: Untreated control group, dexamethasone (DEX; Jiangsu Lianshui Pharmaceutical Co., Ltd., Lianshui, China; $0.5 \mu \mathrm{mol} / \mathrm{l})$, resveratrol (12.5 $\mu \mathrm{mol} / \mathrm{l}$; Sigma-Aldrich, St. Louis, MO, USA) and genistein (25 $\mu \mathrm{mol} / \mathrm{l}$; Sigma-Aldrich) groups. After $1 \mathrm{~h}$ of treatment, $100 \mu \mathrm{l}$ lymphocytes were smeared onto slides for NF- $\mathrm{BB}$ immunocytochemistry staining. After $48 \mathrm{~h}$, the suspension was centrifuged at $400 \mathrm{x}$ g for $10 \mathrm{~min}$ and the supernatant of the lymphocytes was collected, and stored at $-80^{\circ} \mathrm{C}$ for use in TNF- $\alpha$ and MMP-9 assays.

Concentration-effect curves. A kinetic study of the effects of resveratrol and genistein on the proportion of $\mathrm{NF}-\kappa \mathrm{B}$-positive cells, and the secretion of TNF- $\alpha$ and MMP-9 in lymphocytes was performed. Lymphocytes from patients with COPD were treated with $0,3.125,6.25,12.5,25$ and $50 \mu \mathrm{mol} / 1$ resveratrol, and $0,6.25,12.5,25,50$ and $100 \mu \mathrm{mol} / 1$ genistein for $1 \mathrm{~h}$ for NF- $\kappa$ B immunocytochemistry staining, and $48 \mathrm{~h}$ for TNF- $\alpha$ and MMP-9 assays. Concentration-effect curves of NF- $\kappa \mathrm{B}$-positive cell proportion, and TNF- $\alpha$ and MMP-9 concentration following resveratrol and genistein treatment were produced.

Immunocytochemistry. Lymphocytes (100 $\mu 1$ at $2 \times 10^{6}$ density) were smeared on slides and fixed in $4 \%$ paraformaldehyde (Beijing Solarbio Science and Technology Co., Ltd.) for $30 \mathrm{~min}$. Slides were then washed twice for $3 \mathrm{~min}$ in PBS and soaked in 3\% $\mathrm{H}_{2} \mathrm{O}_{2}$ (1:50 dilution; Beijing Haiderun Pharmaceutical Co., Ltd., Beijing, China) for $30 \mathrm{~min}$ to inactivate endogenous peroxidase. After 3 further 3-min washes in PBS, the slides were incubated with $50 \mu$ l goat serum (Beijing Solarbio Science and Technology Co., Ltd.) for $20 \mathrm{~min}$ at room temperature to block non-specific

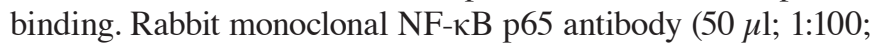


Wuhan Boster Biological Technology Ltd., Wuhan, China; cat. no. PB0321) was then added to the cells and incubated overnight at $4^{\circ} \mathrm{C}$. Slides were washed 3 times in PBS for 3 min and then incubated with monoclonal biotinylated goat anti-rabbit immunoglobulin G (Wuhan Boster Biological Technology Ltd.; cat. no. BA1003) for $20 \mathrm{~min}$ at $37^{\circ} \mathrm{C}$. Slides were washed 3 times in PBS for 3 min and incubated with $50 \mu 1$ streptavidin-peroxidase (SABC kit; Wuhan Boster Biological Technology Ltd.) for $20 \mathrm{~min}$ at $37^{\circ} \mathrm{C}$. The slides were washed 4 times in PBS for 5 min prior to addition of the peroxidase substrate (100 $\mu \mathrm{l}$ 3,3'-diaminobenzidine) for $10 \mathrm{~min}$ at room temperature. The slides were washed in distilled water for 3 min. Finally, the slides were dried naturally, then cleared in xylene (Tianjin Fu Chen Chemical Reagents Factory, Tianjin, China) and mounted in gelatin (Amresco, LLC, Solon, OH, USA). Negative reagent controls were stained in parallel with the primary anti-NF- $\mathrm{\kappa B}$ p65 antibody, and blocked by incubation overnight at $4^{\circ} \mathrm{C}$ with a specific blocking peptide (Wuhan Boster Biological Technology Ltd., Wuhan, China). For each sample, two slides were scored and $\geq 500$ cells were counted randomly on each slide using an Olympus DP71 (Olympus Corporation, Tokyo, Japan). NF-kB p65-positive cells were defined as staining brown in the cellular nucleus and used to calculate the percentage of NF- $\mathrm{kB}$-positive lymphocytes.

Radioimmunoassay. The concentration level of TNF- $\alpha$ in the lymphocyte supernatant was measured using a radioimmunoassay kit (Beijing Chemclin Biotech Co., Ltd., Beijing, China), according to the manufacturer's protocol.

Enzyme-linked immunosorbent assay (ELISA). The concentration level of MMP-9 in the lymphocyte supernatant was measured using an ELISA kit (Wuhan Boster Biological Technology Ltd.), according to the manufacturer's protocol.

Statistical analysis. The data are presented as the mean \pm standard deviation and were analyzed using SPSS software version 14.0 (SPSS, Inc., Chicago, IL, USA). Statistical analysis was conducted using Student's t-test for the independent samples in two groups, analysis of variance and least significant difference test for the comparison of multiple groups and the Pearson linear correlation was used for correlation analysis. $\mathrm{P}<0.05$ was considered to indicate a statistically significant difference.

\section{Results}

$N F-\kappa B, T N F-\alpha$ and MMP-9 signaling pathways are significantly activated in patients with COPD. Compared with healthy subjects, the proportion of NF- $\mathrm{KB}$-positive cells, and the concentrations of TNF- $\alpha$ and MMP-9 in the supernatant were significantly increased in COPD lymphocytes (Table II). Additionally, there was a significant positive correlation between the percentage of NF- $\kappa \mathrm{B}$-positive cells, and the levels of TNF- $\alpha$ and MMP-9 in patients with COPD (Fig. 1).

Concentration-effect curves of resveratrol and genistein in patients with COPD. Compared with control treatment, the percentage of NF- $\kappa \mathrm{B}$-positive cells decreased upon increasing concentrations of resveratrol (3.125-12.5 $\mu \mathrm{mol} / 1 ; \mathrm{P}=0.003$ and
$\mathrm{P}=0.002$, respectively). The proportion of NF- $\mathrm{kB}$-positive cells then gradually increased at 25 and $50 \mu \mathrm{mol} / 1$ resveratrol. The percentage of NF- $\kappa \mathrm{B}$-positive cells was decreased at genistein concentrations of $6.25-25 \mu \mathrm{mol} / 1$, then increased at 50 and $100 \mu \mathrm{mol} / \mathrm{l}$ genistein. The proportion of NF- $\mathrm{kB}$-positive cells significantly decreased compared with the control at 25 and $50 \mu \mathrm{mol} / 1$ genistein $(\mathrm{P}=0.038$ and $\mathrm{P}=0.046$, respectively), with the greatest reduction observed at $25 \mu \mathrm{mol} / 1$ (Fig. 2A). The concentration-effect curves of TNF- $\alpha$ were similar to the percentage of NF- $\mathrm{KB}$-positive cells in the resveratrol and genistein-treated groups (Fig. 2B). Compared with the control, MMP-9 levels decreased with increasing concentrations of resveratrol (3.125-6.25 $\mu \mathrm{mol} / \mathrm{l})$, with resveratrol causing the greatest decrease to MMP-9 levels at $6.25 \mu \mathrm{mol} / 1(\mathrm{P}=0.049)$. MMP-9 levels stabilized at $12.5 \mu \mathrm{mol} / 1$ resveratrol then marginally increased at 25 and $50 \mu \mathrm{mol} / 1$ resveratrol. Additionally, the levels of MMP-9 were gradually decreased by increasing genistein treatment $(6.25-25 \mu \mathrm{mol} / \mathrm{l})$ compared with the control, with the greatest reduction observed at $25 \mu \mathrm{mol} / 1$ genistein $(\mathrm{P}=0.015)$. At higher genistein concentrations, the levels of MMP-9 rose slightly (Fig. 2C). Thus, the optimal concentrations of resveratrol and genistein were 12.5 and $25 \mu \mathrm{mol} / 1$, respectively.

Resveratrol and genistein inhibit the $N F-\kappa B, T N F-\alpha$ and $M M P-9$ signaling pathways. Compared with no treatment, DEX, resveratrol and genistein significantly reduced the percentage of NF- $\mathrm{KB}$-positive cells, and the concentration levels of TNF- $\alpha$ and MMP-9 in lymphocytes from patients with COPD $(\mathrm{P}<0.001)$. The inhibitory effects of resveratrol and genistein were not as potent as DEX, and the effects of resveratrol were significantly greater than those of genistein $(\mathrm{P}<0.001$; Fig. 3). DEX, resveratrol and genistein had no significant effect on NF- $\kappa B$, TNF- $\alpha$ or MMP-9 in the healthy control subjects. Additionally, the present study demonstrated positive correlations between the percentage of NF- $\mathrm{kB}$-positive cells, and the levels of TNF- $\alpha(\mathrm{P}<0.001)$ and MMP-9 $(\mathrm{P}=0.034)$ in the resveratrol-treated group (Fig. 4A and B, respectively), and the genistein-treated group (Fig. 5A and B, respectively) in patients with COPD. A similar trend in correlation curves was observed between Figs. 4 and 5 and Fig. 1.

\section{Discussion}

COPD is associated with chronic inflammation predominantly affecting the lung parenchyma and peripheral airways, resulting in largely irreversible and progressive airflow limitation. This inflammation is characterized by increased levels of alveolar macrophages, neutrophils and $\mathrm{T}$ lymphocytes, which are recruited from the circulation. Oxidative stress is important in driving this inflammation (1). NF- $\mathrm{kB}$ is known to be critical in the regulation of proinflammatory molecules during cellular responses, particularly TNF- $\alpha$, IL- 6 and IL-8.

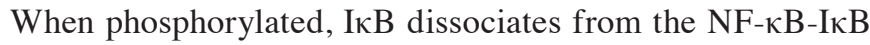
complex, resulting in the translocation of NF- $\kappa \mathrm{B}$ from the cytoplasm to the nucleus. Activation of NF- $\mathrm{KB}$ is regarded as an important initial event in the airway inflammatory response to a variety of stimuli, including infectious agents, toxins, cytokines, growth factors and oxidant stress $(1,5,18)$. $\mathrm{NF}-\mathrm{KB}$ may represent a link between inflammation and 
Table II. Percentage of NF- $\kappa \mathrm{B}$-positive cells, and the levels of TNF- $\alpha$ and MMP-9 in patients with COPD and healthy subjects.

\begin{tabular}{lccc}
\hline Group & NF- $\kappa$ B-positive cells, $\%$ & TNF- $\alpha, \mu \mathrm{g} / 1$ & MMP-9, $\mu \mathrm{g} / \mathrm{l}$ \\
\hline Healthy subjects & $6.5 \pm 2.2$ & $0.89 \pm 0.29$ & $10.21 \pm 2.67$ \\
COPD & $27.9 \pm 6.8^{\mathrm{a}}$ & $2.19 \pm 0.57^{\mathrm{a}}$ & $16.06 \pm 3.60^{\mathrm{a}}$ \\
\hline
\end{tabular}

${ }^{\mathrm{a}} \mathrm{P}<0.01$ vs. healthy subjects. Values are presented as the mean \pm standard deviation. NF- $\kappa \mathrm{B}$, nuclear factor- $\kappa \mathrm{B}$; TNF- $\alpha$, tumor necrosis factor- $\alpha$; MMP-9, matrix metalloproteinase-9; COPD, chronic obstructive pulmonary disease.
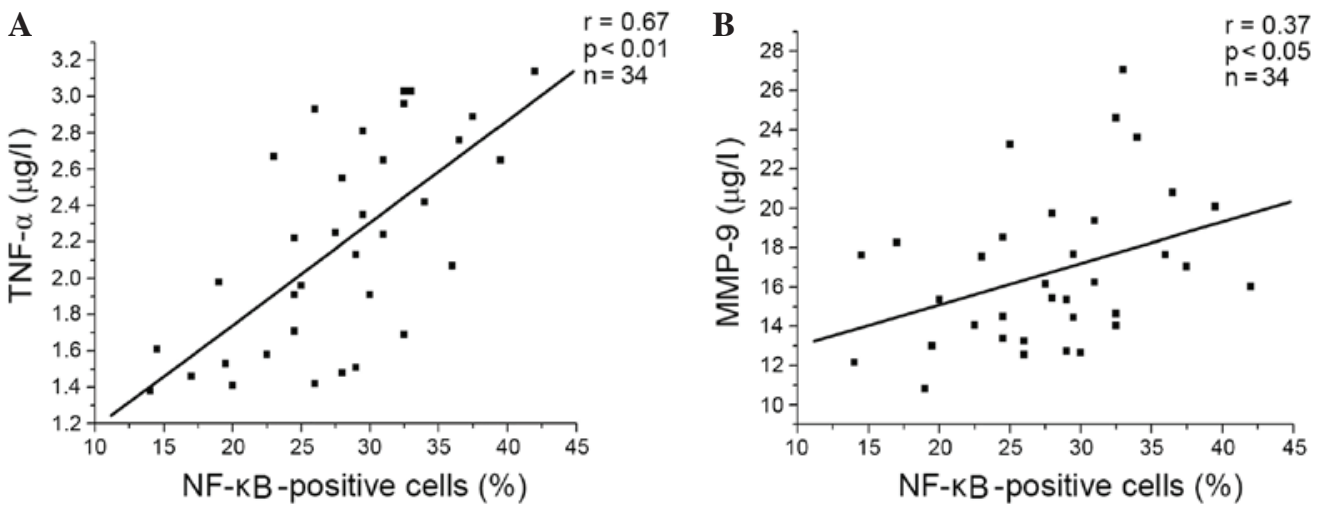

Figure 1. Positive correlations between the percentage of NF- $\kappa \mathrm{B}$ positive cells and the level of $(\mathrm{A}) \mathrm{TNF}-\alpha(\mathrm{r}=0.67, \mathrm{P}<0.01, \mathrm{n}=34)$ and $(\mathrm{B}) \mathrm{MMP}-9$ ( $\mathrm{r}=0.37, \mathrm{P}<0.05$, $\mathrm{n}=34$ ) in patients with chronic obstructive pulmonary disease. TNF- $\alpha$, tumor necrosis factor- $\alpha$; NF- $\kappa \mathrm{B}$, nuclear factor- $\kappa \mathrm{B}$; MMP-9, matrix metalloproteinase-9.

A
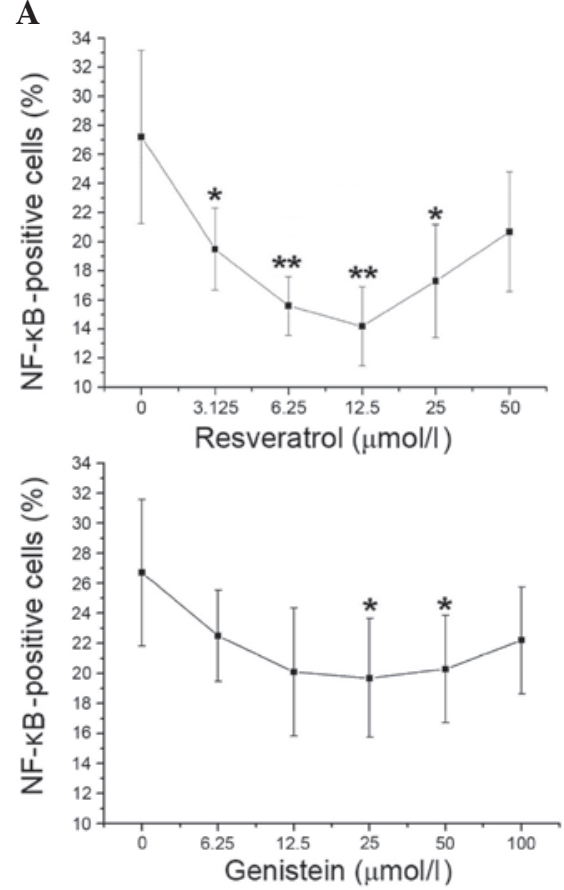

B
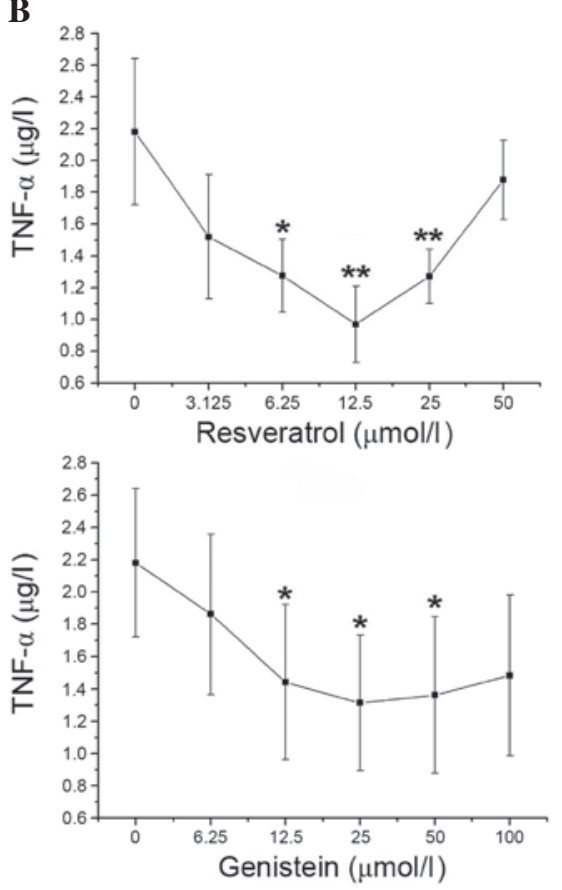

C
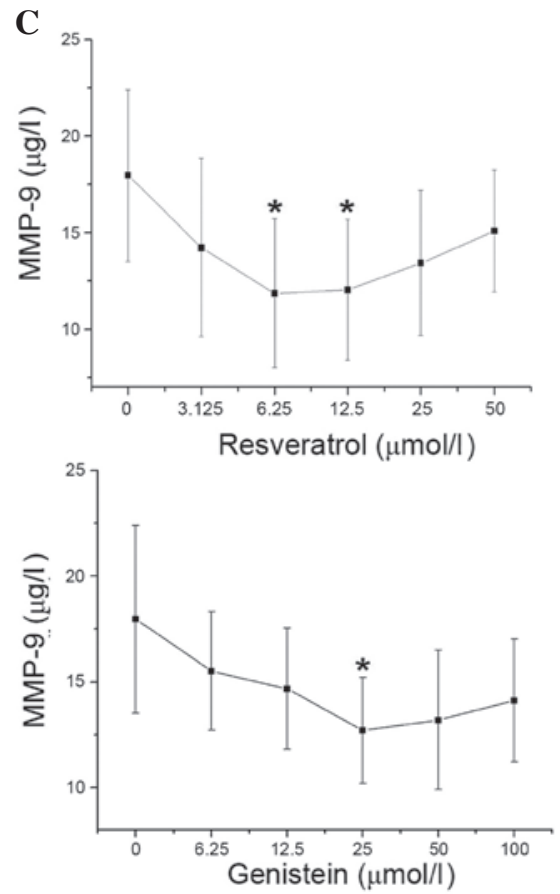

Figure 2. Concentration-effect curves of resveratrol (upper panels) and genistein (lower panels) on the percentage of (A) NF- $\kappa \mathrm{B}$-positive cells, and the levels of (B) TNF- $\alpha$ and (C) MMP-9 in patients with chronic obstructive pulmonary disease. ${ }^{*} \mathrm{P}<0.05$ and ${ }^{* *} \mathrm{P}<0.01 \mathrm{vs}$. $0 \mu$ mol/1 resveratrol or genistein. Values are presented as the mean \pm standard deviation. $n=5$. NF- $\kappa \mathrm{B}$, nuclear factor $\kappa \mathrm{B}$; TNF- $\alpha$, tumor necrosis factor- $\alpha$; MMP-9, matrix metalloproteinase-9.

oxidative stress in chronic inflammatory diseases (19). The data of the present study demonstrated that the percentage of nuclear NF- $\mathrm{BB}$-positive cells in lymphocytes was significantly increased in COPD patients compared with healthy controls. This indicates that translocation and activation of $N F-\kappa B$ is upregulated in patients with COPD. The expression of TNF- $\alpha$, a proinflammatory cytokine, is regulated by NF- $\kappa \mathrm{B}$ and TNF- $\alpha$ activates NF- $\kappa \mathrm{B}$ expression (12). TNF- $\alpha$ has an important amplifying effect in COPD airway inflammation. Furthermore, circulating TNF- $\alpha$ is partially associated with hypoxemia, 

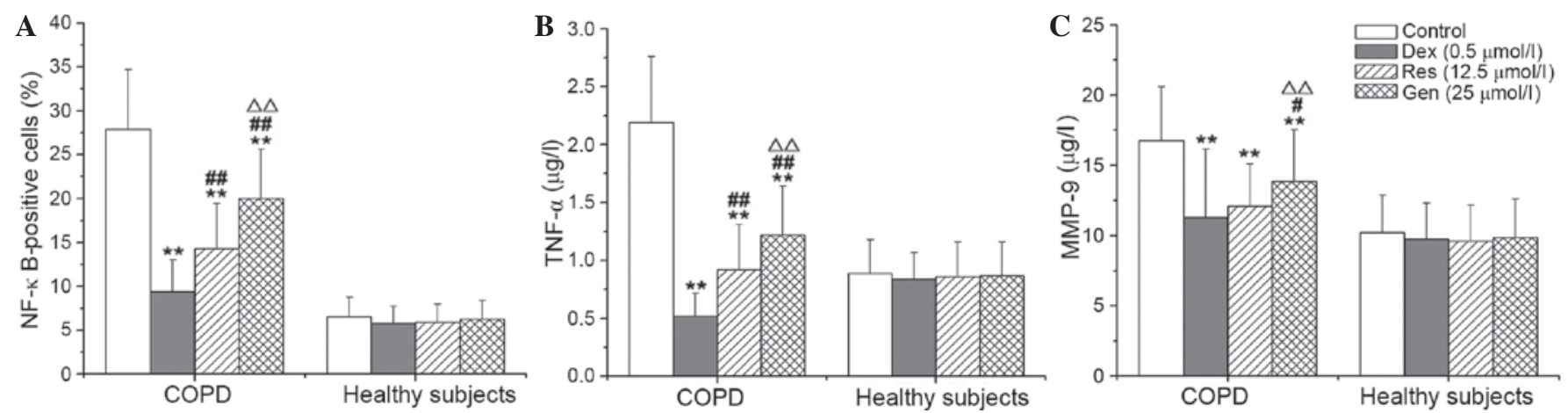

Figure 3. Effects of Res and Gen on (A) NF- $\mathrm{kB}$, (B) TNF- $\alpha$ and (C) MMP-9 in patients with chronic obstructive pulmonary disease. ${ }^{* *} \mathrm{P}<0.01 \mathrm{vs}$. control; ${ }^{\#} \mathrm{P}<0.05$ and ${ }^{\# \#} \mathrm{P}<0.01$ vs. Dex; ${ }^{\Delta \Lambda} \mathrm{P}<0.01$ vs. Res. Values are presented as the mean \pm standard deviation. $\mathrm{n}=34$. NF- $\mathrm{kB}$, nuclear factor- $\kappa \mathrm{B} ; \mathrm{TNF}-\alpha$, tumor necrosis factor- $\alpha$; MMP-9, matrix metalloproteinase-9; Dex, dexamethasone; Res, resveratrol; Gen, genistein.
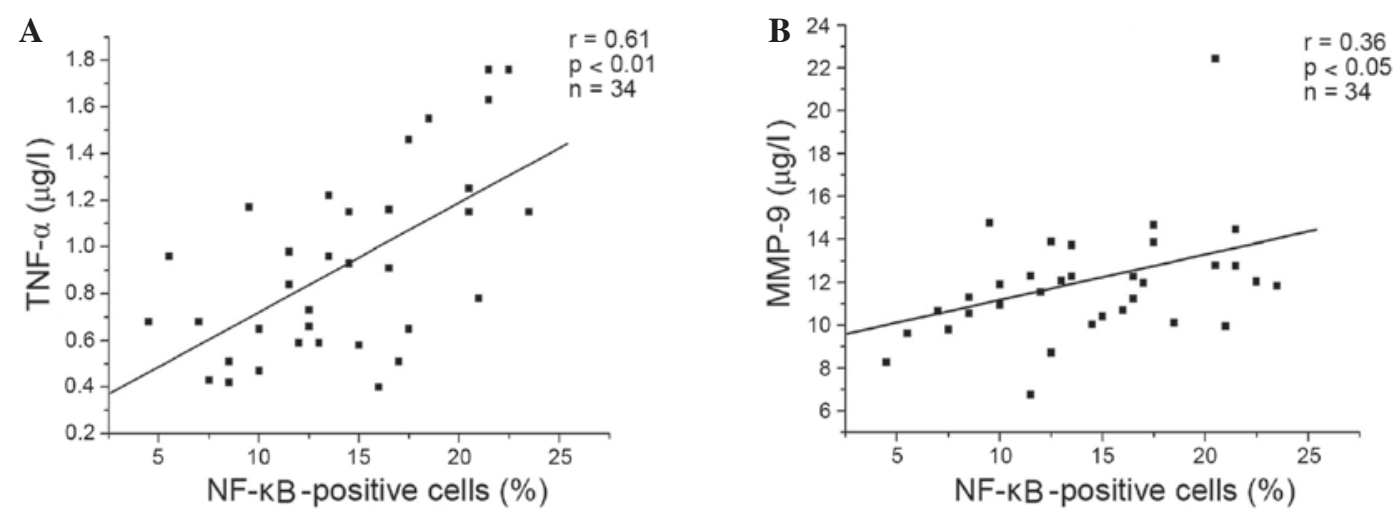

Figure 4. Positive correlations between the percentage of NF- $\mathrm{kB}$ positive cells and the levels of (A) TNF- $\alpha(\mathrm{r}=0.61, \mathrm{P}<0.01, \mathrm{n}=34)$, and (B) MMP-9 ( $r=0.36$, $\mathrm{P}<0.05, \mathrm{n}=34$ ) following resveratrol treatment of lymphocytes from patients with chronic obstructive pulmonary disease. TNF- $\alpha$, tumor necrosis factor- $\alpha$;

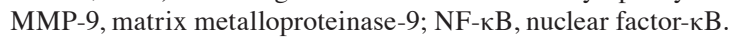
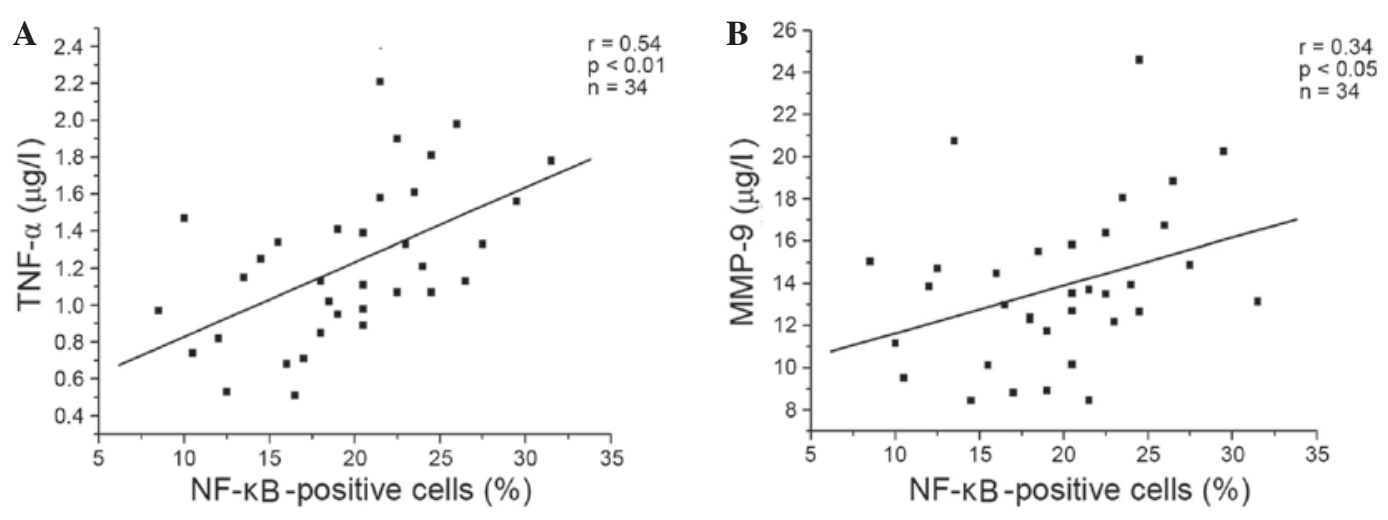

Figure 5. Positive correlations between the percentage of NF- $\mathrm{kB}$ positive cells and the level of (A) TNF- $\alpha(r=0.54, \mathrm{P}<0.01, \mathrm{n}=34)$, and (B) MMP-9 (r=0.34, $\mathrm{P}<0.05, \mathrm{n}=34$ ) following genistein treatment of lymphocytes from patients with chronic obstructive pulmonary disease. TNF- $\alpha$, tumor necrosis factor- $\alpha$;

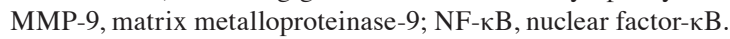

and increased systemic TNF- $\alpha$ levels have previously been demonstrated to be involved in cachexia and skeletal muscle weakness in patients with COPD (1). MMPs are a family of zinc-dependent endopeptidases that are characterized by their ability to degrade components of the ECM. Previous reports have implicated MMPs in numerous physiological and pathophysiological processes, including tissue remodeling, angiogenesis, and cell differentiation and migration. Typically, the enzymatic activity of MMPs is tightly controlled by the secretion of endogenous specific tissue inhibitors of MMPs, however, a disruption of this balance is observed in COPD and other active inflammatory diseases, resulting in tissue injury $(2,20)$. MMP-9 has previously been suggested to be important in the process of COPD and associated with tissue remodeling $(21,22)$. The data of the current study also demonstrated that the levels of TNF- $\alpha$ and MMP-9 were increased 
in the lymphocytes of patients with COPD compared with healthy controls, and there were positive correlations between the percentage of $\mathrm{NF}-\kappa \mathrm{B}$-positive cells, and the concentration of TNF- $\alpha$ and MMP-9 in patients with COPD.

Resveratrol is a polyphenol present in various fruits and vegetables, and is particularly abundant in grapes. The root extract of the weed Polygonum cuspidatum, an important constituent of Japanese and Chinese folk medicine, is also a plentiful source of resveratrol. The anti-inflammatory and antioxidant properties of resveratrol have been previously reported in numerous studies. Knobloch et al (23) demonstrated that resveratrol attenuates the release of inflammatory cytokines from human bronchial smooth muscle cells exposed to lipoteichoic acid in COPD. Birrel et al (24) demonstrated that resveratrol has anti-inflammatory effects in rat lungs challenged with LPS. Furthermore, resveratrol has been demonstrated to be a potent inhibitor of TNF- $\alpha$-induced activation of $N F-\kappa B$, and can suppress TNF- $\alpha$-activated inflammatory pathways in vitro and in vivo (12). The suppression of TNF- $\alpha$ has been observed in normal and tumor cells. Resveratrol also inhibited NF- $\mathrm{BB}$ activation induced by a wide variety of inflammatory agents. The ability of resveratrol to block reactive oxygen species (ROS) generation and lipid peroxidation induced by TNF- $\alpha$ may be associated with its effects on transcription factors and associated kinases. Furthermore, Lee and Kim (25) demonstrated that resveratrol reduces MMP-9 activation through the inhibition of ROS, and the activation of NF- $\kappa \mathrm{B}$ and activator protein-1 in human fibrosarcoma cells. Resveratrol also inhibited TNF- $\alpha$-mediated MMP-9 expression and invasion in human hepatocellular carcinoma cells (26). Knobloch et al (27) reported that resveratrol almost completely reduces MMP-9 release from LPS-exposed alveolar macrophages in patients with COPD. The findings of the present study demonstrate that the activation of NF- $\kappa \mathrm{B}$, and the hypersecretion of TNF- $\alpha$ and MMP-9 in lymphocytes from patients with COPD were inhibited by resveratrol. Additionally, the current study observed that there were positive correlations between the percentage of NF- $\kappa \mathrm{B}$-positive cells, and the concentration levels of TNF- $\alpha$ and MMP-9 following resveratrol treatment of lymphocytes from patients with COPD. This indicates that resveratrol inhibition of TNF- $\alpha$ and MMP-9 may be involved in NF- $\kappa \mathrm{B}$ deactivation.

Genistein, the major isoflavone present in soy, scavenges free radicals, inhibits tyrosine kinases and possesses anti-inflammatory properties. Previous research demonstrated that increased consumption of genistein is associated with improved lung function in patients with asthma (28). Additionally, dietary soy isoflavone supplementation reduces eosinophil leukotriene C4 synthesis and eosinophilic airway inflammation in patients with asthma (29). In a novel finding, Hirayama et al (30) reported that there was a significant positive association between lung function and the intake of isoflavones, including genistein and daidzein. In this previous study, total isoflavone, genistein and daidzein were significantly associated with decreased risk of COPD and breathlessness. Thus, it is likely that isoflavones and their aglycones act as anti-inflammatory agents in the lung and may protect against tobacco carcinogens. Gupta et al (12) reviewed these polyphenols, suggesting that they may protect against pro-inflammatory diseases by downregulation of TNF- $\alpha$ and other proinflammatory biomarkers. Other previous findings indicate that genistein suppresses TNF- $\alpha$-induced inflammation by inhibiting the ROS/Akt/NF- $\kappa$ B pathway and promoting AMP-activated protein kinase activation $(31,32)$. Additionally, Wu et al (33) reported that IL-1 $\beta$-induced upregulation of proMMP-9 was inhibited by genistein in rat brain astrocytes. The data of the present study demonstrated that genistein inhibits the translocation of $N F-\kappa B$ to the nucleus, and decreases the hypersecretion of TNF- $\alpha$ and MMP-9 in lymphocytes from patients with COPD. Additionally, the current study observed that there were positive correlations between the percentage of NF- $\kappa \mathrm{B}$-positive cells, and the levels of TNF- $\alpha$ and MMP-9 following genistein treatment of lymphocytes from patients with COPD. The inhibitory effects of resveratrol and genistein on NF- $\kappa \mathrm{B}$ localization, and TNF- $\alpha$ and MMP-9 levels were not as effective as DEX treatment (a commonly used anti-inflammatory positive control), and genistein was not as effective as resveratrol. The inhibitory actions of resveratrol and genistein on TNF- $\alpha$ were greater that the effects on MMP-9. It is possible that the anti-inflammatory activity of resveratrol and genistein may be more potent than the effects on airway remodeling in patients with COPD.

In conclusion, the present study demonstrated that the translocation of NF- $\kappa \mathrm{B}$ from the cytoplasm to the nucleus, and the secretion of TNF- $\alpha$ and MMP-9 were increased in lymphocytes from patients with COPD compared with healthy controls. Additionally, resveratrol and genistein inhibited the translocation of NF- $\kappa \mathrm{B}$, and decreased TNF- $\alpha$ and MMP-9 concentration levels. Furthermore, the inhibitory actions of resveratrol and genistein on TNF- $\alpha$ were greater than the effects on MMP-9. The results of the present study suggest that resveratrol and genistein may be potential drug candidates for the treatment of COPD.

\section{Acknowledgements}

The present study was supported by The Gansu Healthy Foundation (no. GSWST2010-05) and Gansu Science and Technology Support Program (no. 144FKCA062).

\section{References}

1. Barnes PJ: Cellular and molecular mechanisms of chronic obstructive pulmonary disease. Clin Chest Med 35: 71-86, 2014.

2. Eurlings IM, Dentener MA, Mercken EM, de Cabo R, Bracke KR, Vernooy JH, Wouters EF and Reynaert NL: A comparative study of matrix remodeling in chronic models for COPD; mechanistic insights into the role of TNF- $\alpha$. Am J Physiol Lung Cell Mol Physiol 307: L557-L565, 2014.

3. Mohan D, Gale NS, McEniery CM, Bolton CE, Cockcroft JR, MacNee W, Fuld J, Lomas DA, Calverley PM, Shale DJ, et al; ERICA Consortium: Evaluating the role of inflammation in chronic airways disease: The ERICA study. COPD 11: 552-559, 2014.

4. Kersul AL, Iglesias A, Ríos Á, Noguera A, Forteza A, Serra E, Agustí A and Cosío BG: Molecular mechanisms of inflammation during exacerbations of chronic obstructive pulmonary disease. Arch Bronconeumol 47: 176-183, 2011.

5. Barnes PJ and Karin M: Nuclear factor-kappaB: A pivotal transcription factor in chronic inflammatory diseases. N Engl J Med 336: 1066-1071, 1997.

6. Jayasooriya RG, Dilshara MG, Park SR, Choi YH, Hyun JW, Chang WY, and Kim GY: $18 \beta$-Glycyrrhetinic acid suppresses TNF- $\alpha$ induced matrix metalloproteinase- 9 and vascular endothelial growth factor by suppressing the Akt-dependent NF- $\kappa \mathrm{B}$ pathway. Toxicol In Vitro 28: 751-758, 2014.

7. Tung BT, Rodríguez-Bies E, Talero E, Gamero-Estévez E, Motilva V, Navas P and López-Lluch G: Anti-inflammatory effect of resveratrol in old mice liver. Exp Gerontol 64: 1-7, 2015. 
8. Park EJ and Pezzuto JM: The pharmacology of resveratrol in animals and humans. Biochim Biophys Acta 1852: 1071-1113, 2015.

9. Chen L, Yang S, Zumbrun EE, Guan H, Nagarkatti PS and Nagarkatti M: Resveratrol attenuates lipopolysaccharide-induced acute kidney injury by suppressing inflammation driven by macrophages. Mol Nutr Food Res 59: 853-864, 2015.

10. Chen J, Zhou H, Wang J, Zhang B, Liu F, Huang J, Li J, Lin J, Bai J and Liu R: Therapeutic effects of resveratrol in a mouse model of HDM-induced allergic asthma. Int Immunopharmacol 25: 43-48, 2015.

11. Shao AW, Wu HJ, Chen S, Ammar AB, Zhang JM and Hong Y: Resveratrol attenuates early brain injury after subarachnoid hemorrhage through inhibition of NF- $\kappa \mathrm{B}$-dependent inflammatory/MMP-9 pathway. CNS Neurosci Ther 20: 182-185, 2014

12. Gupta SC, Tyagi AK, Deshmukh-Taskar P, Hinojosa M, Prasad S and Aggarwal BB: Downregulation of tumor necrosis factor and other proinflammatory biomarkers by polyphenols. Arch Biochem Biophys 559: 91-99, 2014.

13. Gao F, Wei D, Bian T, Xie P, Zou J, Mu H, Li J, Lin J, Bai J and Liu R: Genistein attenuated allergic airway inflammation by modulating the transcription factors T-bet, GATA-3 and STAT- 6 in a murine model of asthma. Pharmacology 89: 229-236, 2012.

14. Zhang Y, Dong J, He P, Li W, Zhang Q, Li N and Sun T: Genistein inhibit cytokines or growth factor-induced proliferation and transformation phenotype in fibroblast-like synoviocytes of rheumatoid arthritis. Inflammation 35: 377-387, 2012.

15. Liu XJ, Zhao J and Gu XY: The effects of genistein and puerarin on the activation of nuclear factor-kappaB and the production of tumor necrosis factor-alpha in asthma patients. Pharmazie 65 127-131, 2010.

16. Global Initiative for Chronic Obstructive Lung Disease (GOLD) Global Strategy for the Diagnosis, Management, and Prevention of Chronic Obstructive Pulmonary Disease. www.goldcopd.org. Accessed December 31, 2011.

17. Martin-Piedra MA, Garzon I, Oliveira AC Alfonso-Rodriguez CA, Carriel V, Scionti G and Alaminos M: Cell viability and proliferation capability of long-term human dental pulp stem cell cultures. Cytotherapy 16: 266-277, 2014.

18. Singh R, Mackay AJ, Patel AR, Garcha DS, Kowlessar BS Brill SE, Donnelly LE, Barnes PJ, Donaldson GC and Wedzicha JA: Inflammatory thresholds and the species-specific effects of colonising bacteria in stable chronic obstructive pulmonary disease. Respir Res 15: 114, 2014.

19. Fischer BM, Voynow JA and Ghio AJ: COPD: Balancing oxidants and antioxidants. Int J Chron Obstruct Pulmon Dis 10 261-276, 2015

20. Hirota N and Martin JG: Mechanisms of airway remodeling. Chest 144: 1026-1032, 2013.

21. Yao H, Hwang JW, Sundar IK, Friedman AE, McBurney MW, Guarente L, Gu W, Kinnula VL and Rahman I: SIRT1 redresses the imbalance of tissue inhibitor of matrix metalloproteinase-1 and matrix metalloproteinase-9 in the development of mouse emphysema and human COPD. Am J Physiol Lung Cell Mol Physiol 305: L615-L624, 2013
22. Gross NJ: Novel antiinflammatory therapies for COPD. Chest 142: 1300-1307, 2012.

23. Knobloch J, Wahl C, Feldmann M, Jungck D, Strauch J, Stoelben E and Koch A: Resveratrol attenuates the release of inflammatory cytokines from human bronchial smooth muscle cells exposed to lipoteichoic acid in chronic obstructive pulmonary disease. Basic Clin Pharmacol Toxicol 114: 202-209, 2014.

24. Birrell MA, McCluskie K, Wong S, Donnelly LE, Barnes PJ and Belvisi MG: Resveratrol, an extract of red wine, inhibits lipopolysaccharide induced airway neutrophilia and inflammatory mediators through an NF-kappaB-independent mechanism. FASEB J 19: 840-841, 2005.

25. Lee SJ and Kim M: Resveratrol with antioxidant activity inhibits matrix metalloproteinase via modulation of SIRT1 in human fibrosarcoma cells. Life Sci 88: 465-472, 2011.

26. Yu H, Pan C, Zhao S, Wang Z, Zhang $\mathrm{H}$ and $\mathrm{Wu} \mathrm{W}$ : Resveratrol inhibits tumor necrosis factor-alpha-mediated matrix metalloproteinase-9 expression and invasion of human hepatocellular carcinoma cells. Biomed Pharmacother 62: 366-372, 2008.

27. Knobloch J, Hag H, Jungck D, Urban K and Koch A: Resveratrol impairs the release of steroid-resistant cytokines from bacterial endotoxin-exposed alveolar macrophages in chronic obstructive pulmonary disease. Basic Clin Pharmacol Toxicol 109: 138-143, 2011.

28. Bime C, Wei CY, Holbrook J, Smith LJ and Wise RA: Association of dietary soy genistein intake with lung function and asthma control: A post-hoc analysis of patients enrolled in a prospective multicentre clinical trial. Prim Care Respir J 21: 398-404, 2012

29. Kalhan R, Smith LJ, Nlend MC, Nair A, Hixon JL and Sporn PH: A mechanism of benefit of soy genistein in asthma: Inhibition of eosinophil p38-dependent leukotriene synthesis. Clin Exp Allergy 38: 103-112, 2008.

30. Hirayama F, Lee AH, Binns CW, Hiramatsu N, Mori M and Nishimura K: Dietary intake of isoflavones and polyunsaturated fatty acids associated with lung function, breathlessness and the prevalence of chronic obstructive pulmonary disease: Possible protective effect of traditional Japanese diet. Mol Nutr Food Res 54: 909-917, 2010

31. Li J, Li J, Yue Y, Hu Y, Cheng W, Liu R, Pan X and Zhang P: Genistein suppresses tumor necrosis factor $\alpha$-induced inflammation via modulating reactive oxygen species/Akt/nuclear factor $\kappa \mathrm{B}$ and adenosine monophosphate-activated protein kinase signal pathways in human synoviocyte MH7A cells. Drug Des Devel Ther 8: 315-323, 2014

32. Jeong JW, Lee HH, Han MH, Kim GY, Kim WJ and Choi YH: Anti-inflammatory effects of genistein via suppression of the toll-like receptor 4-mediated signaling pathway in lipopolysaccharide-stimulated BV2 microglia. Chem Biol Interact 212: 30-39, 2014.

33. Wu CY, Hsieh HL, Sun CC, Tseng CP and Yang CM: IL-1 beta induces proMMP-9 expression via c-Src-dependent PDGFR/PI3K/Akt/p300 cascade in rat brain astrocytes. J Neurochem 105: 1499-1512, 2008. 